\title{
Análisis comparativo de los Sistemas de Gestión Ambiental para empresas agroindustriales
}

\author{
Comparative analysis of environmental management systems for \\ agro-enterprises
}

Jennifer K. Castro Camacho' ${ }^{1}$, Néstor-Enrique Cerquera Peña ${ }^{2}$, Alfredo Olaya-Amaya ${ }^{3}$

\begin{abstract}
Resumen
Se desarrolló el análisis comparativo de los sistemas de gestión ambiental propuestos para treinta y cinco empresas dedicadas a actividades agroindustriales, ubicadas en Colombia, Suramérica; estas empresas se clasificaron en veintidós tipos y dos subsectores: alimentario y no alimentario. Se consolidaron los impactos ambientales negativos de los tipos de empresa, obteniéndose un cuadro de impactos generados en todo el sector. Se realizó una comparación de los requisitos de la NTC ISO 14001:2004 para evaluar el estado inicial ambiental de las empresas, obteniéndose una valoración global baja, indicando que las empresas del sector agroindustrial no tienen un compromiso definido respecto al manejo del medio ambiente, que se adelantan algunas acciones puntuales para el control de la contaminación pero es necesario la implementación de los sistemas de gestión formulados, para garantizar un enfoque ambiental integral y el mejoramiento continuo.
\end{abstract}

Palabras clave: Sistemas de gestión ambiental, empresas agroindustriales, NTC ISO 14001:2004, impactos ambientales

\section{Abstract}

A comparative analysis of environmental management systems proposed for thirty-five companies engaged in agribusiness activities, located in Colombia, South America was developed; these companies were classified into twenty-two sub-types: food and non-food. Negative environmental impacts of the types of company were consolidated to give a picture of the impacts generated throughout the sector. A comparison of the requirements of ISO 14001:2004 NTC was conducted to evaluate the initial environmental state companies, yielding a low overall score, indicating that the agribusiness companies have no defined commitment to environmental management, some specific actions to control pollution are ahead but you need to implement management systems formulated to ensure a comprehensive environmental approach and continuous improvement.

Keywords: Environmental management systems, agro-enterprises, NTC ISO 14001:2004

1 Ingeniera Agricola. Estudiante Maestria en Ingenieria y Gestión Ambiental. Universidad Surcolombiana Neiva. Avenida Pastrana Carrera la. Neiva, Huila, Colombia. jeka141285@hotmail.com.

2 Ingeniero Agricola, MSc. en Ingenieria Agricola Universidad Nacional de Colombia, MSc en Dirección Universitaria Universidad de los Andes, Profesor Titular Facultad Ingenieria, Universidad Surcolombiana, Avenida Pastrana Carrera la, Neiva, Huila, Colombia, cerquera@usco.edu.co

3 Licenciado en Biologia y Quimica, $\mathrm{PhD}$ en Ingenieria Área recursos hidráulicos. Profesor Asociado Facultad de Ingenieria. Universidad Surcolombiana Neiva. Avenida Pastrana Carrera la.Neiva, Huila, Colombia. alolaya@usco.educo. 


\section{Introducción}

El sector agroindustrial representa un importante aporte a la contaminación debido a que la mayoria de las industrias descargan altos volúmenes de residuos liquidos, provenientes de sus procesos productivos y del lavado de los equipos, considerados como de alta carga orgánica, con una baja relación de instalaciones para su control. Adicionalmente, existe un importante aporte de desechos sólidos de naturaleza orgánica, generadas en los procesos productivos, así como cartón, papel y plástico en las áreas administrativas y actividades de empaque (Sánchez et al., 2009).

Las agroindustrias de acuerdo a sus actividades desarrolladas se pueden calificar como: (a) de impacto medio en el ambiente y en la salud de acuerdo a la agresividad de sus funciones; (b) de alto impacto en relación al uso de los recursos agua y energia, empleados en los procesos de limpieza, cocción, lavado; $y$; (c) de alto impacto en relación a los altos volúmenes de descargas a la naturaleza de residuos líquidos y desechos sólidos,. Este sector industrial es responsable por más de la mitad de los desechos orgánicos vertidos en el pais, situación que se agrava debido a la poca capacidad de respuestas que tiene el Estado ante la problemática ambiental, lo que evidencia las debilidades que limitan el logro de un desempeño ambiental aceptable (Sánchez et al., 2009).

De acuerdo con Zuluaga y Olarte (2014), el Sistema de Gestión Ambiental -SGA- constituye una de las herramientas de protección ambiental que fortalece la toma de decisiones en el ámbito de políticas, planes, programas y proyectos empresariales, ya que incorpora variables que no han sido consideradas durante los procesos administrativos tradicionales; es el análisis que anticipa los impactos ambientales negativos y positivos de acciones desarrolladas en los diferentes procesos productivos, permitiendo seleccionar las alternativas que maximicen los beneficios y minimicen los impactos no deseados. Su aplicación considera los contextos económicos, sociales e institucionales de la región y del país.
El objetivo de esta investigación fue realizar una sintesis comparativa de los sistemas de gestión ambiental para empresas agroindustriales en Colombia.

\section{Metodología}

Para la recolección de la información se efectuó una revisión bibliográfica de los sistemas de gestión ambiental para empresas que adelantan actividades agroindustriales en Colombia, asi como las Guias ambientales del Ministerio de Ambiente y Desarrollo Sostenible. Se consolidó la información de treinta y cinco empresas que se clasificaron en veintidós tipos y en dos subsectores: alimentario y no alimentario. El estudio adelantado en esta investigación es de tipo descriptivo y de carácter exploratorio.

Se compiló la información correspondiente a los impactos ambientales negativos generados por las actividades de producción de todas las empresas, se asociaron los impactos comunes de acuerdo a la frecuencia de ocurrencia y frecuencia relativa expresada en porcentaje.

La revisión inicial ambiental, en todos los trabajos consultados, fue realizada aplicando la metodología de calificación de cumplimiento de requisitos propuesta por Palom (1997); teniendo en cuenta esto, se promediaron las calificaciones obtenidas para cada requisito y para la valoración global de todas las empresas, con lo que se obtuvo el grado de satisfacción o grado de cumplimiento de los requisitos de la Norma NTC ISO 14001:2004; lo que se considera clave en la implementación de un sistema de gestión ambiental para el sector agroindustrial.

\section{Resultados}

\subsection{Tipos de empresas}

Las empresas estudiadas se clasificaron en veintidós tipos, y estos a su vez se dividieron en dos subsectores: alimentario y no alimentario. En la 
Tabla 1. Clasificación de las empresas del subsector alimentario

\begin{tabular}{|c|c|c|c|}
\hline Tipo Empresa & Cantidad & Subsector & Fuente \\
\hline Industria $\mathrm{Arro} Z$ & 2 & & Borbón y Moya, 1997; Cabrera y Camacho, 2008 \\
\hline Piscicola & 2 & & Diaz y Trujillo, 2012; Joaqui, 2011 \\
\hline Derivados lácteos & 3 & & $\begin{array}{l}\text { Buyucué y Tovar, 2008; Medellín 2007; Bermúdez, } \\
2009\end{array}$ \\
\hline Avicola (huevos y pollos) & 2 & & Bolaños y Camargo, 2011; Muñoz, 2012 \\
\hline Chocolate & 1 & & González, 2009 \\
\hline Industria café & 3 & Alimentario & $\begin{array}{l}\text { Ramírez, 2013; Herrera y Valencia, 2005; Cardona } \\
\text { y Gómez, } 2012\end{array}$ \\
\hline Frutas y verduras & 1 & & Gutiérrez et al, 2009 \\
\hline $\begin{array}{l}\text { Plantas de beneficio de carne } \\
\text { y derivados cárnicos }\end{array}$ & 4 & & Mas, 2013; Lara, 2006; Echeverri, 2009; Mejí 2011 \\
\hline Porcicola & 1 & & Machado et al, 2010 \\
\hline Panelero & 1 & & Mejia, 2007 \\
\hline Cerveza & 1 & & Parada, 2010 \\
\hline Azúcar & 1 & & Ruiz y Sandino, 2013 \\
\hline
\end{tabular}

tabla 1 se presentan las empresas correspondientes al subsector alimentario, así como la fuente bibliográfica que permitió la consolidación de la información.

En la figura 1 se puede observar el porcentaje de cada uno de los tipos de empresa del subsector alimentario que han formulado su sistema de gestión ambiental. En el subsector alimentario, las "plantas de beneficio de carne y derivados cárnicos" son las que más han reportado la implementación de un sistema de gestión ambiental con una participación del $18 \%$, esto se debe principalmente a que la cadena de la carne en Colombia se caracteriza por una compleja interacción en sus procesos productivos, $y$ de estas actividades se generan muchos subproductos que requieren la implementación inmediata de programas de manejo ambiental (SIC, 2014). En segundo lugar, se tiene a las empresas de "derivados lácteos" y la "industria del café" con un 14\%; en los otros tipos de empresa la implementación varía de un $9 \%$ a un $4 \%$. En general, las empresas del subsector deben trabajar más en la implementación de estos sistemas de gestión ambiental para garantizar el mejoramiento continuo de las mismas.

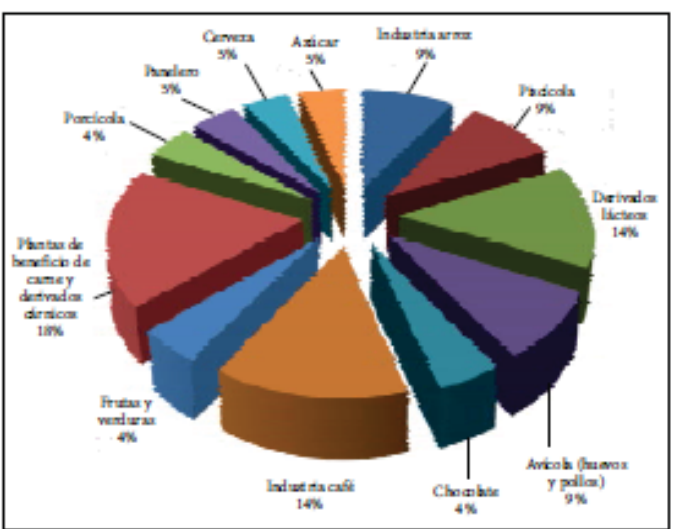

Figura 1. Tipos de empresa del subsector alimentario con sistemas de gestión ambiental.

La industria bananera, correspondiente al tipo de empresa "frutas y verduras", no reporta sistemas de gestión ambiental, sin embargo Minambiente (2014) formuló una guia ambiental para la agroindustria de banano para su implementación 
Tabla 2. Clasificación de las empresas objeto de estudio del subsector no alimentario

\begin{tabular}{|c|c|c|c|}
\hline Tipo Empresa & Cantidad & Subsector & Fuente \\
\hline Madera & 2 & \multirow{10}{*}{ No alimentario } & Pérez y Upegui, 2008; Jaramillo, 2013 \\
\hline Flores & 3 & & Álvarez et al, 2007; Londono, 2003; Berrocal y Salazar, 2004 \\
\hline Palma & 1 & & Enriquez, 2009 \\
\hline Centros de acopio & 1 & & Calpa y López, 2008 \\
\hline Algodón & 1 & & Mateus, 2012 \\
\hline Grasas y aceites & 1 & & Comisión Nacional del medio ambiente, 1998 \\
\hline Biocombustibles & 1 & & Araujo y Misle, 2008 \\
\hline Curtiembres & 1 & & Murcia, 2013 \\
\hline Tabaco & 1 & & Alfaro, 2011 \\
\hline $\begin{array}{l}\text { Alimentos balanceados } \\
\text { para animales }\end{array}$ & 1 & & León, 2009 \\
\hline
\end{tabular}

en los procesos industriales con fines de exportación.

En la tabla 2 se presenta la clasificación de las empresas correspondientes al subsector no alimentario que fueron analizadas e igualmente la fuente bibliográfica.

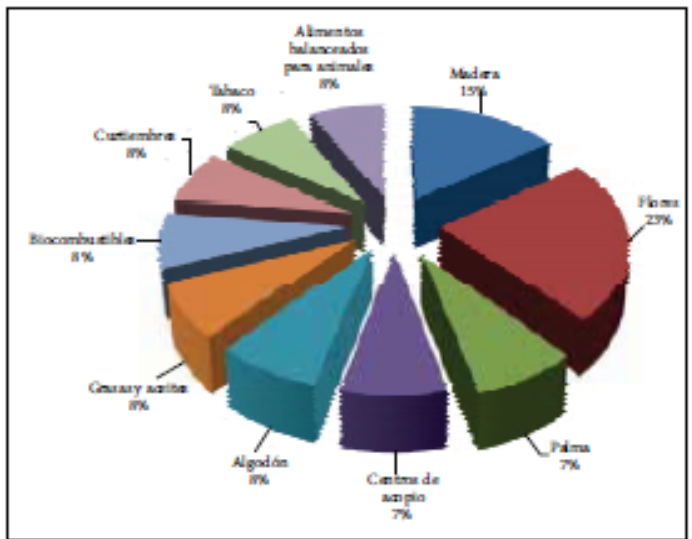

Figura 2. Tipos de empresa subsector no alimentario con sistemas de gestión ambiental

La figura 2 muestra los tipos de empresas del subsector no alimentario que han formulado su sistema de gestión ambiental. Las actividades en las empresas dedicadas a la "poscosecha de flores" se realiza con altos estandares de calidad que garantizan la existencia de programas sociales y medioambientales en todos los cultivos del pais (Proexport, 2014), y esto se refleja siendo el tipo de empresa que registra el mayor porcentaje de implementación de sistemas de gestión ambiental, que corresponde al 23\%. El segundo lugar en la formulación del sistemas de gestión ambiental corresponde al tipo de empresa "Madera" con un $15 \%$, y los otros tipos de empresas varian entre un $7 \%$ y $8 \%$.

\subsection{Impactos ambientales generados por las empresas del sector agroindustrial}

Según Cabrera y Camacho, 2008, los aspectos ambientales son los diferentes elementos, actividades y productos de la empresa que pueden interactuar con el medio ambiente. Los trabajos de investigación consultados hicieron la identificación de los aspectos ambientales de las empresas y la evaluación de los impactos ambientales asociados con los procesos industriales, que desarrollan dichas empresas, utilizando metodologias como: "Matriz de Leopold", "redes de interacción" y "reconocimiento de campo". Los impactos ambientales negativos generados en las empresas, su frecuencia de ocurrencia y la frecuencia relativa expresada en porcentaje, se muestran a continuación en la tabla 3: 
Tabla 3. Impactos ambientales negativos generados en los tipos de empresa del sector agroindustrial

\begin{tabular}{clrc}
\hline \multirow{2}{*}{ Item } & Impactos Negativos & $\begin{array}{c}\text { Frecuencia de } \\
\text { ocurrencia }\end{array}$ & $\%$ \\
\hline 1 & Contaminación del aire por material particulado y gases & 17 & 77,27 \\
\hline 2 & Generación de residuos sólidos ordinarios y especiales & 15 & 68,18 \\
\hline 3 & Contaminación por ruido & 14 & 63,64 \\
\hline 4 & Contaminación del recursos hidrico: alteración de parámetros fisicoquimicos & 13 & 59,09 \\
\hline 5 & Riesgo laboral y deterioro de la salud & 11 & 50,00 \\
\hline 6 & Vertimiento de aguas residuales y otros & 9 & 40,91 \\
\hline 7 & Generación de olores desagradables & 31,82 \\
\hline 8 & Proliferación de plagas: roedores e insectos & 7 & 27,27 \\
\hline 9 & Deterioro del paisaje & 6 & 27,27 \\
\hline 10 & Modificación del hábitat, alteración de fauna y flora & 22,73 \\
\hline 11 & Disminución del caudal y deterioro en los cuerpos de agua & 5 & 18,18 \\
\hline 12 & Desperdicio del recurso hidrico & 4 & 18,18 \\
\hline 13 & Altos consumos de energía eléctrica & 4 & 18,18 \\
\hline 14 & Afectación de vías por aumento de flujo vehicular & 4 & 13,64 \\
\hline 15 & Erosión y degradación de las caracteristicas fisico químicas del suelo & 3 & 13,64 \\
\hline 16 & Generación de alta carga contaminante por pelambre* & 3 & 4,55 \\
\hline 17 & Afectación de las aguas por curtido al cromo* & 1 & 4,55 \\
\hline 18 & Residuos sólidos con cromo* & 4,55 \\
\hline 19 & Afectación del agua por presencia de grasas, ácidos orgánicos y anilinas* & 4,55 \\
\hline *Impacto presente en un solo tupo de empresa pero que requtere un control especial. & 1 &
\end{tabular}

El impacto ambiental que se presenta con mayor frecuencia en las empresas agroindustriales es la "contaminación del aire por material particulado y gases", que se reporta en el $77,27 \%$ de ellas. Este impacto se da por las emisiones de particulas en los procesos de transformación como la trilla, por la combustión de los motores de los equipos de las plantas y por chimeneas.

La "generación de residuos sólidos ordinarios y especiales" se presenta en el $68,18 \%$ de las empresas. Las causas de este impacto están asociadas con la generación de residuos que influyen negativamente en la estética del paisaje y en la contaminación del suelo.

El 63,64\% de las empresas, consideran a la "contaminación por ruido" como un impacto muy desfavorable debido a que afecta las funciones auditivas en las personas que laboran en ellas y que afecta a los habitantes del área de influencia de las plantas.

Otro impacto que incide negativamente en las empresas y su interacción con el medio ambiente es la "contaminación del recursos hídrico: alteración de parámetros fisicoquimicos" con un $59.09 \%$ de ocurrencia. El consumo de agua en algunas empresas es significativo. El uso principal que las empresas le dan al agua es para adelantar las labores de limpieza y desinfección, y se descargan directamente a los cuerpos de agua, alterando su calidad y disponibilidad para usos primarios.

El "riesgo laboral y deterioro de la salud" que es considerado negativo por el $50 \%$ de los tipos de empresa, se debe a las actividades propias de las plantas de procesamiento, generalmente en el área de transformación que implique maquinaria 

con partes móviles y que interactúan de manera directa con los trabajadores.

En la figura 3 se observa que los cinco impactos analizados arriba presentan una frecuencia de ocurrencia mayor al cincuenta por ciento, siendo estos los impactos ambientales significativos a los que se les debe prestar mayor atención para la formulación de programas, metas y objetivos del sistema de gestión ambiental de empresas agroindustriales.

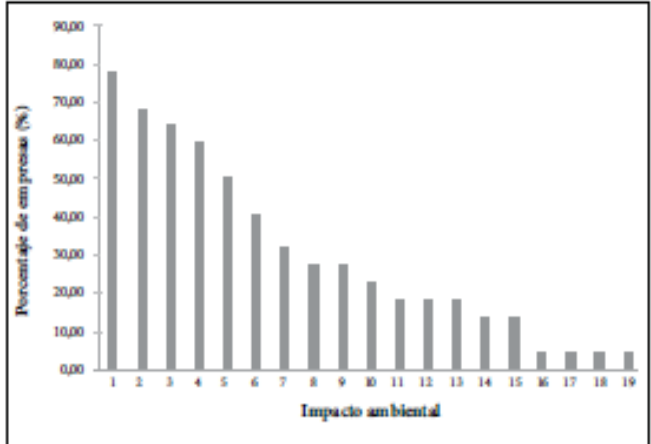

Figura 3. Impactos ambientales negativos ordenados de acuerdo al porcentaje de empresas que lo generan

Los impactos ambientales "vertimiento de aguas residuales y otros", "generación de olores desagradables", "proliferación de plagas: roedores e insectos", "deterioro del paisaje", "modificación del hábitat, alteración de fauna y flora", "disminución del caudal y deterioro en los cuerpos de agua", "desperdicio del recurso hidrico", "altos consumos de energía eléctrica" y "erosión y degradación de las características fisico quimicas del suelo", tienen una incidencia que varia desde el $40,91 \%$ hasta el $13,64 \%$. Estos impactos, de menor frecuencia de ocurrencia, se pueden asociar al diseño de los objetivos y metas ambientales para cada empresa asi como a los indicadores del sistema de gestión ambiental.

En cuanto a los impactos "generación de alta carga contaminante por pelambre", "afectación de las aguas por curtido al cromo", "residuos sólidos con cromo", "afectación del agua por presencia de grasas, ácidos orgánicos y anilinas", son impactos muy especiales del tipo de empresa dedicada a "curtiembre". En estos procesos las aguas utilizadas sufren alteraciones en su $\mathrm{pH}$, es decir, dicha agua contiene una gran variedad de sustancias que traen como consecuencia contaminación al medio ambiente, estas aguas no son aptas para el consumo humano, porque generan diversas enfermedades virales, respiratorias, inmunológicas, cutáneas, entre otras; además se deteriora la fauna aledaña a las fuentes hidricas (Murcia, 2013).

\subsection{Evaluación de los requisitos NTC ISO 14001:2004 de los sistemas de gestión am- biental del sector agroindustrial}

En la figura 4 se puede apreciar la valoración promedio del grado de satisfacción de los requisitos de la NTC ISO 14001:2004 obtenidas por las empresas del sector agroindustrial, que aplicaron la metodologia propuesta por Palom (1997).

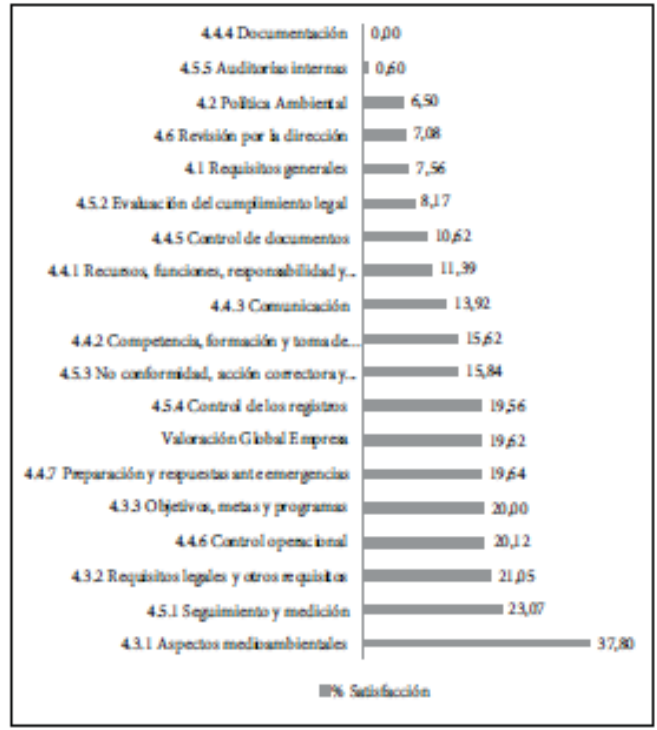

Figura 4. Histograma ordenado de acuerdo al grado de satisfacción de los requisitos de la NTC ISO 14001:2004 presentes en el sector agroindustrial.

Teniendo como base la información obtenida de las empresas del sector agroindustrial (Figura 4), a continuación se presenta el grado de satisfac- 
clón de los requisitos de la NTC ISO 14001:2004, de acuerdo a lo reportado en las revisiones ambientales iniciales consultadas e igualmente la apreclación del respectivo requlsito.

Requisitos generales: Las empresas del sector agrolndustrlal están empezando a tomar medıdas para definir y documentar un sistema de gestlón ambiental en ellas. Su calıficación es de 7,56. Por lo tanto es urgente insistir ante las gerencias sobre la importancla del tema ambiental, para fortalecer su compromiso con la implementación de un sistema de gestlón ambiental que permita el mejoramiento continuo.

Politica ambiental: Su calıficación es de 6,50. Esto indica que algunas de las empresas están defintendo los principlos de acción o las directrices generales sobre el medio amblente, que permitan fijar los objetivos y metas ambientales.

Aspectos ambientales: Este estudio permite ev1denclar que este requisito tlene un grado de satisfacción del 37,80\% el cual, según Palom (1997), corresponde a "minima medida" con tendencla a "grado aceptable". Las empresas tienen metodologias claras que permiten fijar directrices para el manejo de los impactos amblentales, algunos tipos de empresas cuentan con las guias amblentales aportadas por el Ministerlo de Amblente y Desarrollo Sostenible. Es importante resaltar que este requisito presentó una calificación por enclma de la obtenida en la "Valoración Global de las empresas" que llegó a un 19,62\%, lo que evidencla que el sector agroindustrlal tlene un interés manifiesto en la implementación de los Sistemas de Gestión Amblental.

Requisitos legales y otros requisitos: Con un grado de satısfacción del $21,05 \%$, este requisito es uno de los que más se acerca al cumplimlento de "minima medıda". Conforme a lo estipulado en la Norma NTC ISO 14001:2004, toda empresa debe tener un procedimiento que permita identificar y tener acceso a los requisitos legales y otros que influyan directa o indirectamente y que tengan aplicabilidad en los aspectos ambientales de sus

Tabla 4. Requisitos legales y otros aplicables al sector agrolndustrial

\begin{tabular}{|c|c|}
\hline $\begin{array}{l}\text { Impacto ambiental significativo u } \\
\text { otro componente ambiental }\end{array}$ & Disposición legal de ámbito nacional, regional o local \\
\hline $\begin{array}{l}\text { Contaminactión del aire por } \\
\text { material particulado y gases }\end{array}$ & $\begin{array}{l}\text { Decreto } 2811 \text { del } 18 \text { de diciembre de } 1974 \text {, Decreto } 02 \text { de } 1982 \text {, Ley } 30 \text { de } 5 \\
\text { de marzo de } 1990 \text {, Decreto } 948 \text { del } 5 \text { de junio de } 1995 \text {, Decreto } 2107 \text { del } 30 \text { de } \\
\text { noviembre de } 1995 \text {, Resolución } 1351 \text { del } 14 \text { de dictembre de } 1995 \text {, Resolución } \\
619 \text { del } 7 \text { de Julıo de } 1997 \text {, Resoluctón } 1048 \text { del } 6 \text { de dictembre de } 1999 \text {, Ley } \\
629 \text { del } 27 \text { de diciembre de } 2000 \text {, Resoluctón } 0068 \text { del } 18 \text { de enero del } 2001 \text { y } \\
\text { Resolución } 0601 \text { del } 4 \text { de abril del } 2006 \text {. }\end{array}$ \\
\hline $\begin{array}{l}\text { Generactón de residuos sólidos } \\
\text { ordınarios y especiales }\end{array}$ & $\begin{array}{l}\text { Decreto } 1715 \text { del } 4 \text { de agosto de } 1978 \text {, Ley } 09 \text { del } 24 \text { de enero de 1979, Resolu- } \\
\text { ctón } 541 \text { del } 14 \text { de diclembre de } 1994 \text { y Decreto } 1713 \text { del } 6 \text { de agosto de } 2002 \text {. }\end{array}$ \\
\hline Contaminactón por ruldo & $\begin{array}{l}\text { Decreto } 2811 \text { del } 18 \text { de diclembre de } 1974 \text {, Resolución } 08321 \text { del } 4 \text { de agosto de } \\
1983 \text { y Resolución } 0627 \text { del } 7 \text { de abril de } 2006 \text {. }\end{array}$ \\
\hline $\begin{array}{l}\text { Contaminactón del recursos } \\
\text { hidrico: alteractón de parámetros } \\
\text { fistcoquimicos }\end{array}$ & $\begin{array}{l}\text { Constitución politica de Colombla } 1991 \text {, Ley } 23 \text { de } 1973 \text {, Decreto } 2811 \text { del } 18 \\
\text { de diclembre de } 1974 \text {, Ley } 09 \text { del } 24 \text { de enero de } 1979 \text {, Decreto } 1594 \text { de } 1984, \\
\text { Decreto } 3930 \text { del } 25 \text { de octubre de } 2010 \text {, Decreto } 4728 \text { del } 23 \text { de diciembre de } \\
2010 \text {, Resoluctón } 1096 \text { del } 17 \text { de noviembre de } 2000 \text {, Decreto } 3039 \text { de } 2010 \text {, } \\
\text { Decreto } 1575 \text { del } 9 \text { de mayo de } 2007 \text {, Resolución } 2115 \text { del } 22 \text { de junto de } 2007 \text {, } \\
\text { Resolución } 1618 \text { del } 7 \text { de mayo de } 2010 \text { y Decreto } 2667 \text { del } 21 \text { de diciembre de } \\
2012 \text {. }\end{array}$ \\
\hline $\begin{array}{l}\text { Ruesgo laboral y deterioro } \\
\text { de la salud }\end{array}$ & $\begin{array}{l}\text { Resolución } 08321 \text { del } 4 \text { de agosto de 1983, Decreto } 1295 \text { del } 22 \text { de junto de } 1994 \\
\text { y Ley } 1562 \text { del } 11 \text { de julto de } 2012 \text {. }\end{array}$ \\
\hline Industria & Decreto 1299 del 22 de enero de 2008 \\
\hline
\end{tabular}


actividades, productos y servicios. En la tabla 4 se muestra los requisitos legales y otros aplicables al sector agroindustrial derivados de los impactos amblentales más significattvos encontrados en esta investigación.

Objetivos, metas y programas: El requisito fue valorado con un cumplımiento del $20 \%$. Ésto debido a que algunas empresas, aunque han realizado evaluación de impacto ambiental, todavia les falta fortalecer los objettivos, metas y programas que conlleven a un compromiso real por parte de todo el personal que labora en ellas.

Recursos, funciones, responsabilidad y autoridad: Su grado de satısfacción fue del 11,39\%, lo que se interpreta como la existencia de algunas funclones de responsabilidad amblental; se debe trabajar en la elaboración de un manual de funclones que permita generar responsabilidades y evidenclas sobre el cumplimlento de las metas y objetivos.

Competencia, formación y toma de conciencia: La valoración de este requisito es de 15,62\%. En el sector agroindustrial se hacen algunas capacitaciones relacionadas con el medio ambiente, pero es necesario generar conclencla en el personal mediante diversas actividades y responsabilidades que le permitan hacer parte importante de un proceso que garantice su trabajo en armonia con el amblente.

Comunicación: Se obtuvo un grado de satısfacclón del $13,92 \%$. Es necesario que las empresas implementen estrateglas de comunicación de los programas y proyectos ambientales, asi como procedımlentos para establecer y mantener comunicaciones internas entre las dıferentes áreas $\mathrm{y}$ funclones de las empresas.

Documentación: El grado de satısfacción de este requisito es de $0 \%$, lo que indica que "no existe en ninguna medida". Las empresas no cuentan con un documento que recopile la información sobre temas y programas amblentales proplos.
Control de documentos: El requisito control de documentos fue valorado con un $10,62 \%$ de satısfacción, lo cual muestra la necesidad de implementar procedimientos claros en cuanto al manejo del archivo en las empresas y la implementación de un adecuado sistema de control y manejo de la documentación.

Control operacional: Este requisito se acerca a la valoración "minima medida", con un $20.12 \%$; se documentan sólo los trámites administratıvos. Para mejorar este requisito se debe documentar los procedimientos operativos y crear procedimientos para actividades de carácter ambiental especificamente.

Preparación $y$ respuesta ante emergencias: Este requisito tlene un grado de satısfacción del $19,64 \%$, lo que indica que las empresas no thenen claramente definidos los procedimlentos de emergenclas amblentales.

Seguimiento y medición: Este requisito se acerca a la valoracıón "mintma medida" con un $23,07 \%$, no obstante, se debe trabajar en la elaboración de procedimientos documentados que permitan medir y controlar las características medıoamblentales significattvas.

Evaluación del cumplimiento legal: Este requlsito obtuvo una valoración de $8,17 \%$, ya que en las empresas existe interés por el cumplimiento legal pero no existen procedimientos para el monitoreo periódico del cumplimiento a la normatividad.

No conformidad, acción correctiva y acción preventiva: Las empresas tlenen estudios de impacto amblental en donde se proponen algunas acciones correctlvas y preventivas, por ello este requisito se calıficó con $15,84 \%$, aunque se deben elaborar los procedimlentos y métodos para determinar las fallas o deficiencias del sistema y las respectivas acclones correctivas y preventivas.

Control de registros: El control de reglstros thene un grado de sattsfacción del $19,56 \%$, las em- 
presas cuentan con archlvos de registros de estudios amblentales $\mathrm{y}$ permisos amblentales, pero deben establecer procedimientos para identificar, archivar y elıminar registros amblentales.

Auditoria interna: En las empresas del sector agrolndustrial prácticamente "no existe en ninguna medida" las auditorias de calıdad, por ello su calificacıón es de $0,60 \%$, sólo se hacen audıtorias contables.

Revisión por la gerencia: La gerencla no cuenta con procedimientos establecidos para las revislones, se realizan revisiones de documentos y se tienen en cuenta los temas medioambientales, pero no hay evidencla de la ruta que siguen dichos documentos, por ello la valoración es $7,08 \%$.

Valoración global del sector: La valoraclón global promedıo de las empresas del sector agroindustrial es de $19,62 \%$, lo que indica que se está empezando a trabajar en las medidas para la implementación de un sistema de gestión amblental con el fin de mejorar su responsabilidad amblental.

\section{Conclusiones}

Los tupos de empresa que más se han preocupado por el diseño e implementación de un sistema de gestıón amblental en el sector alımentarıo son las "plantas de beneficlo y derivados cárntcos", "derlvados lácteos" e "Industrla del cafê". En el sector no alımentarlo la "Industrla de las flores" es la que más ha realızado sıstemas de gestión ambiental. Es importante la implementación del sistema de gestlón amblental ya que cada vez exısten mayores niveles de competencla a nivel internaclonal, a los que deberán enfrentarse las empresas agroindustriales producto de convenlos o tratados que exigen la protección del medıo amblente.

Los impactos amblentales negatıvos más significattvos para el sector agrolndustrial son: "contaminación del alre por materıal partıculado y gases", "la generacıón de residuos sólıdos ordinarlos y especiales", "contaminación por ruldo", "contaminación del recurso hidrıco" y "rlesgo laboral y deterioro de la salud". Para su manejo y control, se deben fijar lineamentos en la politica ambiental, objetivos y metas amblentales en el sistema de gestıón amblental.

La valoracıón global promedio de la revisı́n amblental Inicial mostró que las empresas del sector agrolndustrial no ttenen un compromlso definldo respecto al manejo del medio amblente, aunque adelantan algunas acclones puntuales para el control de la contaminación. Esta valoración evidencla la necesidad de un compromiso real de los directivos para la implementación de los sistemas de gestıón formulados, que garantıce un enfoque amblental por parte de las empresas. De igual manera y como parte del mejoramiento continuo se debe realızar perı́dicamente la verıficación de cumplimiento de cada requisito de la NTC ISO 14001 para evaluar la eficacla de la puesta en marcha de los sistemas de gestıón amblental.

\section{Referencias bibliográficas}

1. Alfaro, E. 2011. Estudio de impacto ambtental Tabacalera Andina S.A. Documento empresartal de Tanasa (Duran). Guayaqul

2. Álvarez, C., Acevedo, J., Hernández, C., Piedrahita, S. 2007. Gestıón y Certificacıón Agroamblental: camino a la sustentablidad de la floricultura. Revista Producción más Limpia, vol. 2, No. 1, 67-90p.

3. Araujo, E., Misle, P. 2008. Propuesta para la adopctón de un Ststema de Gestıón Ambtental en empresas productoras de etanol. Revista Voces: Tecnologia y pensamtento, vol. 2 , No. 1-2, 23-47p.

4. Bermúdez, C. 2009. Sustema de Gestión Ambtental ISO 14004 para una empresa de producctón lechera en la Sabana de Bogotá. Trabajo de grado para optar al Titulo de Administrador de empresas agropecuarias. Programa de administractón agropecuarla. Untverstdad de La Salle. Bogotá D.C.

5. Berrocal, L., Salazar, J. 2004. Diseño de un Sistema de Gestión Ambiental basado en la Norma ISO 14001:96 para los restduos sólidos generados por el proceso de producción del clavel mintatura en el culttvo "Flores de Funza S.A.C.I". Trabajo de grado para optar al Título de Ingeniero Industrial. Facultad de Ingenteria. Pontficicia Universidad Javeriana. Bogotá D.C. 
6. Bolaǹos, D., Camargo, M. 2011. Diseǹo del Ststema de Gestión Ambiental para la avicola La Dominga. Trabajo de grado para optar al Titulo de Especialista en Ingenteria Amblental. Facultad de Ingenteria. Universidad Surcolombtana. Nelva

7. Borbón, R.A., Moya, L. 1997. Propuesta de un ststema de gestión ambiental para la industria Molinera de arroz en el departamento del Hulla. Trabajo de grado para optar al Titulo de Especialısta en Ingenieria Ambtental. Facultad de Ingentería. Untverstdad Surcolombiana. Netva.

8. Buyucué, C., Tovar, M. 2004. Sistema de gestión ambiental para la fabrica Quesos Retma del munlctpto de Tarqui-Hulla. Trabajo de grado para optar al Titulo de Espectalista en Ingenieria Ambiental. Facultad de Ingenteria. Universidad Surcolombiana. Netva.

9. Cabrera, C., Camacho, O. 2008. Ststema de Gestıón Amblental para la Agroindustria de arroz Inverstones PTC S.A. Trabajo de grado para optar al Título de Especialista en Ingenieria Ambiental. Facultad de Ingenieria. Untversidad Surcolomblana. Netva.

10. Calpa, J., López, D. A. 2008. Formulación del plan de manejo ambiental para la Planta de Acopio de Alimentos del Valle "AL.IVAL. S.A.". Trabajo de grado para optar al Titulo de Espectalista en Ingenieria Ambtental Local. Facultad de Clenctas Ambientales. Universidad Tecnológıca de Peretra Sede San Juan de Pasto. San Juan de Pasto.

11. Cardona, N., Gómez, O. 2012. Propuesta de modelo de integración entre los eslabones que participan en la cadena productiva y comercial del sector cafetero risaraldense para aumentar su competittvidad en el mercado internacional. Trabajo de grado para optar al Título de Administrador de empresas. Facultad de Cienclas económicas y administrativas. Universidad Católica de Pereira. Pereira.

12. Comısıón Nacıonal del Medı́o Ambiente. 1998. Fabricación de grasas $y$ aceltes vegetales $y$ subproductos. Guia para el control y prevención de la contaminación industrial. Santlago, Pp 59.

13. Diaz, N., Trujlllo, F. 2012. Sistema de Gest1ón Ambtental para la Pıscicola Castalıa Ltda. Garzón, Hulla Colombia. Trabajo de grado para optar al Título de Especialista en Ingenieria Ambiental. Facultad de Ingenieria. Untversidad Surcolomblana. Netva.

14. Echeverri, J. 2009. Gestión Ambiental en la empresa Cárnicos y Alımentos S.A. Trabajo de grado para optar al Titulo de Ingentera ambiental. Facultad de
Ingenleria amblental. Corporactón Untversitarıa Lasallista. Caldas, Antıoquia

15. Enriquez, L.M. 2009. Estrateglas para la implementación de la Norma ISO 14001 en empresas productoras de palma de aceite de la Zona Ortental Colombtana. Trabajo de grado para optar al Título de Magister en Gestión Ambiental. Facultad de estudios ambientales y rurales. Ponttificia Universidad Javerıana. Bogotá D.C.

16. González, E. 2009. Propuesta para la creacıón del departamento de gestión ambiental para la compañia Occidental de Chocolates S.A. municipio de Palermo-Hulla. Trabajo de grado para optar al Titulo de Espectalista en Ingenieria Ambiental. Facultad de Ingenieria. Universidad Surcolombiana. Netva.

17. Guttérrez, S, Gil, J., Álvarez, C. 2009. Implementación de un plan integral de residuos sólidos generados en el proceso de producción en una industrla alımenticta de salsas y conservas de ptina. Revista Producctón más L.ımpla, vol. 4, No. 2, 30-43p.

18. Herrera, B., Valencia, D. 2005. Desarrollo de las actividades del plan de manejo ambiental- parte aire- de la trilladora San Mateo del munictpio de Chinchiná. Trabajo de grado para optar al Título de Especialısta en Ingenieria Ambiental con énfasis en Sanitarla. Facultad de Ingenleria y Arquitectura. Untversidad Nacional de Colombta Sede Manizales. Manizales

19. Instituto Colombtano de Normas y Técnicas y Certuficación. 2004. Sistemas de Gestión Ambiental: Requisitos con orientación para su uso. NTC ISO 14001:2004. Santafé de Bogotá

20. Jaramillo, A. 2013. Formulación del Sistema de Gesttón Ambiental en la empresa J.A.M. Maderas en el municipio de Dosquebradas. Trabajo de grado optar al Titulo de Admintstrador Ambiental. Facultad de Clenctas Ambientales. Untversidad Tecnológica de Pereira. Pereira.

21. Joaqui, S. 2011. Gestlón Amblental para sistemas productivos piscicolas, en ecoststemas altoandinos en el contexto de la producción más limpia. Revista ambiente y sostenibulidad., vol. 1, 18-24p.

22. Lara, M. 2006. Sistemas de Gestión Ambtental en Mataderos Munictpales Caso: Matadero de lagunillas-FRISUCRE. Trabajo de grado para optar al Titulo de Magister en Gestlón de Recursos naturales y medio amblente. Centro de Interamericano de Investigactón en Ambtente y Territorto. Universtidad de los Andes. Mérida. 
23. León, K. V. 2009. Implementación de un Sistema de Gestión Ambiental para la Empresa ITALCOL. S.C.A. Informe final de Práctica empresarıal para optar al Título de Ingeniera Ambiental. Facultad de Ingentería y Administración. Universidad Ponttificla Bolivariana. Pledecuesta.

24. Londoño, C. X. 2003. Revistón Ambiental Inictal y planeación de un Ststema de Gestión Ambiental para el cultivo de rosas Comercializadora Internacional San Luis Ltda. Trabajo de grado para optar al Título de Especialista en Ingenjeria Ambtental. Facultad de Ingenteria. Universidad de la Sabana. Chia.

25. Machado, E., Robayo, Y., Ayala, H., Chávez, A. 2010. Plan de manejo ambtental para un proyecto Porcicola. Estudio de caso: Proyecto La Zambera, Otanche, Boyaca. Revista gestión integral en ingeniería neogranadina, vol. 2, No. 2, Pp 14.

26. Mas, M. I. 2013. Propuesta de implantación de un Ststema de Gestıón Ambłental, en un matadero de ganado ovino y vacuno en Gatblel, según la Norma UNE-EN-ISO 14001:2004. Trabajo final de carrera. Untverstdad Politécnica de Valencla. Programa de Licenclatura en Clenclas Amblentales. España.

27. Mateus, A. 2012. Mejoramiento de la productiv1dad de la hilatura del algodón y su proyección textil, desde el enfoque de la producción más limpia y el LCA. Trabajo para optar al Titulo de Magister en Ingenieria con énfasis en Ingenieria Industrial. Facultad de Ingenteria. Universidad Nacional de Colombia. Bogotá D.C.

28. Medellin, F. 20007. Diseño de una guía para la implementación de un ststema de gestión ambiental según la Norma Técnica Colombiana NTC-ISO 14001:2004 para el sector de lácteos caso Planta Funza Colanta. Trabajo de grado para optar al Titulo de Ingeniera ambiental y sanitarias. Facultad de Ingenieria ambiental y sanitaria. Universidad de La Salle. Bogotá D.C.

29. Mejia, A. M. 2007. Propuesta para la implementacón del Sistema de Gestlón Ambiental en el Trapiche Panelero- HVC. Documento final de Práctica empresartal para optar al Titulo de Administrador del Medio Amblente. Facultad de Clenclas Ambientales. Universidad Tecnológica de Pereira. Pereira.

30. Mejia, J. 2011. Optimización del Sıstema de Gestuón Ambiental en la planta de derivados cárnico Colanta. Trabajo de grado para optar al Titulo de Ingentera ambtental. Facultad de Ingenteria am- biental. Corporación Universitarta Lasallısta. Caldas, Antioquia

31. Ministerio de Ambiente y desarrollo sostentble. Consultado el 01 de mayo de 2014. http://www.mlnamblente.gov.co/documentos/Sector_Bananero. pdf

32. Muñoz, A. C. 2012. Actualızación del Ststema de Gestión Ambtental Alımentos FIKO S.A.S. Trabajo de grado para optar al Titulo de Ingeniera ambiental. Facultad de Ingenieria ambiental. Corporactón Universitaria Lasallista. Caldas, Antioquia

33. Murcla, E. R. 2013. Estrateglas para la mejora de los Sistemas de Gestión Ambiental en las industrias de las curtiembres en el munictpto Villa Pinzón Cundınamarca. Trabajo de grado para optar al Titulo de Contador Público. Facultad de Ciencias Económicas. Untversidad Milıtar Nueva Granada. Bogotả D.C.

34. Palom, O. 1997. ISO 14001: La Nueva Auditorla Medio Ambiental paso a paso, software. Barcelona: Gestıón y Planificación Integral. s.f. 78p.

35. Parada, J.A. 2010. Implementación del Sistema de Gestión Amblental de Bavaria S.A. Cerveceria de Bucaramanga con base en la Norma ISO 14001:2004. Trabajo de grado para optar al Titulo de Ingentera ambiental. Facultad de Ingenteria. Untversidad Ponttficta Bolivartana. Bucaramanga

36. Pérez, M., Upegui, G. 2008. Diseño de un Sistema de gestıón ambiental para la empresa forestal Putumayo S.A. ubicado en el municipio de Villagarzón, departamento del Putumayo. Trabajo de grado para optar al Titulo de Especialista en Ingenteria Ambtental. Facultad de Ingenteria. Untversidad Surcolombiana. Netva.

37. Proexport. Oportunidades de negocto en el sector flores y plantas vivas. Consultado el 30 de abril de 2014. http $/ /$ www.proexport.com.co/node/ 1242

38. Ramirez, A. 2013. Mejoramiento continuo en el Sistema de Gestión de ALEXCAFE S.A.S. Trabajo de grado para optar al Titulo de Administrador de empresas. Facultad de Clenclas económicas y administrativas. Untwersidad Católica de Peretra. Pereira.

39. Ruiz, S., Sandino, M. 2013. Propuesta metodológica para la gestıón de restduos en la industria azucarero usando lean manifacturing. Magazín empresarial Economia y Empresa., vol. 9, No. 21, 45-57p.

40. Sánchez, R., Najul, M., Brito, E., Ferrara, G. 2009. El Manejo de los Residuos Sólidos en la Industria de 
Agroalimentos en Venezuela. Revista Interctencla, vol. 34 , No. 2.

41. Zuluaga, A., Olarte, J. 2014. Ststema de gestión ambtental para agrotndustrias colombianas. Consul- tado el 30 de abril de 2014. http://es.scribdcom/ doc/55696207/Ststema-de-Gestion-Ambiental-para-Agroindustrlas-Colomblanas 